\title{
Predicting stroke outcome: what does the 'stroke prognostocrit level' indicate?
}

\author{
Ralph L Sacco
}

A 55-year-old woman with acute onset of speech difficulty and right hemiparesis is brought within 1 hour of symptom onset to the emergency department of a local primary stroke center. She undergoes standard stroke protocol assessment, and receives intravenous thrombolysis. An urgent 'stroke prognostocrit level' is drawn, and the results indicate that she has an elevated risk of stroke progression and neurological deterioration. She is consequently treated with a new agent shown to be of value in patients at high risk of worsening.

This might sound like science fiction, but reliable prognostic biomarkers to classify stroke patients more accurately into high-risk or lowrisk categories might be within reach. The goals are to quantify probabilities of various outcomes, identify patients needing closer observation, and possibly select patients for the best treatment approaches. As neurological health-care providers, we often rely on various clinical symptoms and signs, severity scores, sociodemographic features, comorbid features, vascular risk factors, imaging characteristics, and etiopathological classifications to help us predict outcomes. The features that are most predictive often depend on the outcome of interest. Among stroke patients, relevant outcomes include death, worsening, hemorrhagic transformation, recurrence, disability, and quality of life. Multidimensional clinical models, such as the Stroke Prognostic Instrument, have been developed to estimate risks of stroke or death and to guide secondary stroke prevention (Kernan W et al. [2000] Stroke 31: 456-620). Such approaches might be useful in the counseling of patients and families about prognosis, and to guide health-care providers regarding treatment, although further studies of their utility are needed.

In the Practice Points section of this issue, three articles regarding the use of serum markers as aids to the estimation of prognosis after stroke are reviewed. Elevated serum
... . reliable

prognostic

biomarkers to

classify stroke

patients more

accurately

into high-risk

or low-risk

categories

might be within

reach

RL Sacco is Associate Chair of Neurology and Professor of

Neurology and

Epidemiology, and

Director of the Stroke and Critical Care

Division at Columbia

University and the

New York Presbyterian

Hospital, New York,

NY, USA, and an

Advisory Board

member of Nature

Clinical Practice

Neurology.

\section{Competing interests}

The author has declared

associations with the

following companies:

Boehringer Ingelheim,

GlaxoSmithKline, Merck,

Sanofi-BMS and Wyeth.

See the article online for full

details of the relationship.

www.nature.com/clinicalpractice doi:10.1038/ncpneuro0345 levels of high-sensitivity C-reactive protein (hsCRP) helped to identify a greater mortality risk at 3 months in acute ischemic stroke patients treated with tissue plasminogen activator (tPA). Elevated fibrin degradation product D-dimer levels helped to predict which acute stroke patients had the greatest risk of early neurological deterioration. Moreover, D-dimer levels aided the identification of patients with a less favorable prognosis after surgery for aneurysmal subarachnoid hemorrhage. All three of these studies, and their respective Practice Point commentaries, emphasize the need for replication and validation before we can adopt these serum markers into clinical practice.

A stroke prognostic test should be sensitive and specific, reproducible, fairly easy to conduct in a standard fashion, and relatively inexpensive, and should add predictive value to other clinical measures. Such approaches have been used to evaluate the predictive value of hsCRP in cardiovascular risk prediction, but no consensus has been reached on whether this test should be more widely utilized (Cook NR et al. [2006] Ann Intern Med 145: 21-29). Recent studies have also indicated that the Lp-PLA 2 (lipoprotein-associated phospholipase $A_{2}$ ) assay might be helpful for ascertaining stroke risk. Future prognostic tests are likely to harness the power of genetics: early identification of changes in molecular expression profiles might provide prognostic value, just as they have done with certain cancers (Fan C et al. [2006] N Engl J Med 355: 560-569).

At present, we do not have the ideal 'stroke prognostocrit level' to guide therapeutic decisions. Potential markers have been identified, and other candidates are likely to be discovered. Further adequately powered prospective studies are needed to clarify the importance of these putative serum markers-and possibly genes-as clinically meaningful prognostic predictors with additive predictive value above what can already be determined clinically. 